\title{
Critical Perioperative Evaluation of Diabetes Mellitus: A Key
}

\section{to Avoid Unnecessary Complications}

\author{
Srivastava $\mathbf{P}^{1 *}$ and Srivastava $\mathrm{S}^{2}$ \\ ${ }^{1}$ Department of Surgery, Om Surgical Center \& Maternity Home, Varanasi, India \\ 2Department of obstetrics and gynaecology, Om Surgical Center \& Maternity Home, \\ Varanasi, India
}

Editorial

Volume 2 Issue 3

Received Date: August 25, 2018

Published Date: September 04, 2018

*Corresponding author: Pankaj Srivastava, Laparoscopic, Thoracic, Thoracoscopic \& VATS Surgeon, Om Surgical Center \& Maternity Home, India, Tel: +91-542-2586191; Email: drpankajbns@gmail.com

\section{Editorial}

\section{We cannot solve our problem with the same thinking we used when we created them...!!}

Famous scientist Albert Einstein aptly quoted this thought and it is very well applicable to all dimensions of science including medicine. Critical evaluation of the problem is the solution of that problem. Diabetes mellitus is a syndrome that involves each cell of the human body and it imparts its effect on that too. The diabetic patients have been appreciated as having increased risk of most commonly surgical site infection and other adverse outcome leads to increased morbidity and increased hospital stay thereby increasing inpatient costs. The usual causes of all are hypo and hyperglycemia, multiple associated diseases like cardiovascular, neurological, misuse or inappropriate use of insulin, use of multiple hypoglycemic agents etc. Good glycemic control decreases the morbidity in diabetics and nondiabetic as well. The perioperative mortality rate is reported to be up to $50 \%$ higher than that of the non-diabetic population [1].

Good glycemic control has a great impact on the risk of post-operative infection across a variety of surgical specialities [1]. The National Inpatient Diabetes Survey found that not only hyperglycemia but hypoglycemia also deteriorates the patient's condition. Approximately 25\% of patients on surgical wards experienced a hypoglycemic event and inpatient hypoglycemia is associated with increased mortality [2]. Few postoperative deaths have also been reported due to diabetic ketoacidosis [3]. Cardiovascular diseases like hypertension, coronary artery disease and stroke have been associated two to four folds in established diabetics and may lead to multiple postoperative complications [4]. Many surgical patients with diabetes are commonly associated with these cardiovascular diseases and microvascular disease like nephropathy or neuropathy. Fluid overload is a major threat for patients with impaired cardiac function and/or nephropathy. Post-operative cardiac arrhythmias are also common in diabetes, particularly in those with autonomic dysfunction or a prolonged QTc interval [5]. The postoperative hypotension is another complication, related to a combination of autonomic dysfunction, inadequate fluid replacement and inadequate monitoring of hypotensive therapies, may precipitate renal failure in those with nephropathy and hypotensive falls in the elderly. It has been found that physicians often fall short to identify high-risk patients before surgery and do not ensure that appropriate perioperative interventions are provided [6]. Proper identification and management of high-risk population might lead to substantial improvements in outcomes and thereby decreasing the postoperative deaths. It has been recommended that putting an identifier in the medical record for all patients with diabetes admitted to hospital may improve the management $[7,8]$. Not all hospitals have comprehensive guidelines for management of glycemia in inpatients, and many lack a strategy for achieving good glycemic control [3]. Proper knowledge and understanding of diabetes and its management is poor amongst both medical and paramedical staff.

The surgical procedures in diabetics should be planned in the morning hours to avoid unnecessary prolonged fasting which in turn disturbs the blood sugar 


\section{Open Access Journal of Endocrinology}

level. The patients who are on insulin therapy, or having poorly controlled sugar levels, or taking many oral hypoglycemic agents (OHA), or unable to resume proper diet; an insulin-glucose infusion is the best way of maintaining their normal blood sugar levels postoperatively. Previously these patients were treated by traditional "sliding scale insulin" but it was found ineffective. Patients who are already taking insulin should receive an insulin-glucose infusion either prior to or at the time of induction of anesthesia (or by $1000 \mathrm{hrs}$ at the latest) and the infusion should be continued for 24 hours post-operatively or until the patient is eating adequately. Patients who are never treated by insulin but receiving more than one type of OHA and undergoing major surgery should be managed with an insulin-glucose infusion for the first 24 hours post-operatively.

The anesthetist must be informed regarding the presence of autonomic neuropathy as this impairs cardiovascular reflexes that may result in hypotension at the induction of anesthesia, as well as an impaired respiratory drive post-operatively. Surgery may also precipitate myocardial ischemia. The presence of significant coronary artery disease may not always result in typical symptoms of ischemic heart disease. Silent myocardial ischemia may be present in up to $20 \%$ of patients with diabetes, especially those with macrovascular disease elsewhere, with microalbuminuria or with 2 or more additional cardiovascular risk factors. Such patients should undergo cardiac evaluation prior to major surgery. Dehydration and nephrotoxic drugs must be avoided in patients with diabetic nephropathy whereas the dose of other medications may need to be altered because of changes in drug clearance.

With proper education, diabetic patients should be allowed to maintain control on their medication. They should be directed for the proper use, dosing and maintenance of their blood sugar levels on their own. Many patients have several years experience and be expert in self-medication. The aim of surgical team and paramedics should be to avoid hypoglycemia or hyperglycemia during the fasting and perioperative period, until the patient is eating and drinking normally. The postoperative diet plan, fluid management, oral and intravenous intake, blood glucose management plan, and any alterations to existing medications, should be clearly be mentioned on the postoperative orders and clarified to the paramedical staff. After recovery from anesthesia, the patient should also be communicated for their medicines, diet and postoperative management plan. If subcutaneous insulin is required in non-insulin patients, or any change in the type of insulin or the timings of doses, the concerned specialist diabetes team should be contacted for advice.

Perioperative hyperglycemia may lead to postoperative sepsis [9], endothelial dysfunction [10], cerebral ischemia [11], impaired wound healing [12,13] diabetic ketoacidosis [14], and hyperglycemic hyperosmolar syndrome [15]. It has been found that values of HbA1c value less than $6.5 \%$ or more than $9.0 \%$ may be associated with increased mortality within one year in clinical type 2 diabetes [16]. We have also advocated even monthly HbA1c measurement in chronic diabetics for better blood glucose control and avoid any complication in time [17]. Glycemic monitoring in the perioperative setting is very crucial because unrecognized hypoglycemia is more dangerous than hyperglycemia as it may result in a number of neurological complications including somnolence, unconsciousness, convulsions, altered sensorium and even death [18].

Conclusively, proper preoperative patient's evaluation, extensive investigations, thorough blood glucose monitoring and judicious use of insulin alleviate the complications associated with diabetic patient undergoing surgical procedure.

\section{References}

1. Frisch A, Chandra P, Smiley D, Peng L, Rizzo M, et al. (2010) Prevalence and clinical outcome of hyperglycemia in the perioperative period in noncardiac surgery. Diabetes Care 33(8): 1783-1788.

2. Rayman G Inpatient audit. Diabetes Update.

3. Hamblin PS, Topliss DJ, Chosich N, Lording DW, Stockigt JR (1989) Deaths associated with diabetic ketoacidosis and hyperosmolar coma. 1973-1988. Med J Aust 151(8): 441-442,444.

4. Stamler J, Vaccaro O, Neaton JD, Wentworth D (1993) Diabetes, other risk factors, and 12-yr cardiovascular mortality for men screened in the multiple risk factor intervention trial. Diabetes Care 16(2): 434-444.

5. Veglio M, Chinaglia A, Cavallo-Perin P (2004) QT interval, cardiovascular risk factors and risk of death in diabetes. J Endocrinol Invest 27(2): 175-181.

6. Cullinane M, Gray AJ, Hargraves CM, Lansdown M, Martin C, et al. (2003) Who operates when? II: the 2003 report of the National Confidential Enquiry into Perioperative Deaths. 


\section{Open Access Journal of Endocrinology}

7. Moghissi ES, Korytkowski MT, Dinardo MM, Hellman R, Hirsch IB, et al. (2009) American Association of Clinical Endocrinologists and American Diabetes Association consensus statement on inpatient glycemic control. Diabetes Care 32(6): 1119-1131.

8. NHS Institute for Innovation and Improvement. Think glucose: inpatient care for people with diabetes.

9. Rayfield EJ, Ault MJ, Keusch GT, Brothers MJ, Nechemias C, et al. (1982) Infection and diabetes: the case for glucose control. Am J Med 72(3): 439-450.

10. Hempel A, Maasch C, Heintze U, Lindschau C, Dietz R, et al. (1997) High glucose concentrations increase endothelial cell permeability via activation of protein kinase $C \alpha$. Circ Res 81(3): 363-371.

11. Pulsinelli WA, Levy DE, Sigsbee B, Scherer P, Plum F (1983) Increased damage after ischemic stroke in patients with hyperglycemia with or without established diabetes mellitus. Am J Med 74(4): 540544.

12. Marhoffer W, Stein M, Maeser E, Federlin K (1992) Impairment of polymorph nuclear leukocyte function and metabolic control of diabetes. Diabetes Care 15(2): 256-260.
13. McMurry JF (1984) Wound healing with diabetes mellitus. Better glucose control for better wound healing in diabetes. Surg Clin North Am 64(4): 769778.

14. Walker M, Marshall SM, Alberti KGMM (1989) Clinical aspects of diabetic ketoacidosis. Diabetes Metab Rev 5(8): 651-663.

15. Brenner WI, Lansky Z, Engelman RM, Stahl WM (1973) Hyperosomolar coma in surgical patients: an iatrogenic disease of increasing incidence. Annals of Surgery 178(5): 651-654.

16. Nicholas J, Charlton J, Dregan A, Gulliford MC (2013) Recent HbA1c values and mortality risk in type 2 diabetes. Population based case-control study. PLoS ONE 8(7): e68008.

17. Srivastava P, Srivastava S, Srivastava R (2018) HbA1c-Glycated Hemoglobin: Perfect Tool to Curb the Diabetes. J Endocrinol 2(2): 1-3.

18. Turchin A, Matheny ME, Shubina M, Scanlon SV, Greenwood B, et al. (2009) Hypoglycemia and clinical outcomes in patients with diabetes hospitalized in the general ward. Diabetes Care 32(7): 1153-1157. 